\title{
Next updates of atmospheric correction processor Sen2Cor
}

Pflug, Bringfried, Louis, Jérôme, Debaecker, Vincent, Müller-Wilm, Uwe, Quang, Carine, et al.

Bringfried Pflug, Jérôme Louis, Vincent Debaecker, Uwe Müller-Wilm, Carine Quang, Ferran Gascon, Valentina Boccia, "Next updates of atmospheric correction processor Sen2Cor," Proc. SPIE 11533, Image and Signal Processing for Remote Sensing XXVI, 1153304 (20 September 2020); doi: $10.1117 / 12.2574035$

SPIE. Event: SPIE Remote Sensing, 2020, Online Only 


\title{
Next updates of atmospheric correction processor Sen2Cor
}

\author{
Bringfried Pflug*a , Jérôme Louis ${ }^{\mathrm{b}}$, Vincent Debaecker ${ }^{\mathrm{b}}$, Uwe Mueller-Wilm ${ }^{\mathrm{c}}$, Carine Quang ${ }^{\mathrm{d}}$, Ferran \\ Gascon $^{\mathrm{e}}$, Valentina Boccia ${ }^{\mathrm{e}}$ \\ ${ }^{a}$ German Aerospace Centre, Remote Sensing Technology Institute, Rutherfordstr. 2, 12489 Berlin- \\ Adlershof, (Germany), Email: bringfried.pflug@dlr.d; ${ }^{b}$ Telespazio France - A Leonardo / Thales \\ Company, 26 Av. JF Champollion, 31023 Toulouse Cedex 1(France), Email: \\ jerome.louis@telespazio.com, vincent.debaecker@telespazio.com; ${ }^{\mathrm{C}} \mathrm{TPZV}-\mathrm{D}$ - Telespazio Vega \\ Deutschland - A Leonardo / Thales Company Email: Uwe.mueller-wilm@telespazio-vega.de; ${ }^{d} \mathrm{CS}$ \\ Group, France, Email: carine.quang@csgroup.eu; ${ }^{\mathrm{e}}$ European Space Agency, ESRIN, Italy Email: \\ Ferran.gascon@esa.int,Valentina.Boccia@esa.int
}

\begin{abstract}
The Sentinel-2 mission is dedicated to land monitoring, emergency management and security. It serves for monitoring of land-cover change and biophysical variables related to agriculture and forestry. The mission is also used to monitor coastal and inland waters and is useful for risk and disaster mapping. The Sentinel-2 mission is fully operating since June 2017 with a constellation of two polar orbiting satellite units. Both Sentinel-2A and Sentinel-2B are equipped with an optical imaging sensor MSI (Multi-Spectral Instrument) which acquires optical data products with spatial resolution up to $10 \mathrm{~m}$.

Accurate atmospheric correction of satellite observations is a precondition for the development and delivery of high quality applications. Therefore the atmospheric correction processor Sen2Cor was developed with the objective of delivering land surface reflectance products. Sen2Cor is designed to process monotemporal single tile Level-1C products, providing Level-2A surface (Bottom-of-Atmosphere) reflectance product together with Aerosol Optical Thickness (AOT), Water Vapour (WV) estimation maps and a Scene Classification (SCL) map for further processing.

The paper will give an overview of the Level-2A product content and up-to-date information about the data quality of the Level-2A products generated with Sen2Cor 2.8 in terms of Cloud Screening and Atmospheric Correction. In addition the paper gives an outlook on the next updates of Sen2Cor and their impact on Level-2A Data Quality.
\end{abstract}

Keywords: atmospheric correction, cloud masking, Sentinel-2, Sen2Cor, performance assessment, data quality, Level$2 \mathrm{~A}$ product

\section{INTRODUCTION}

The Copernicus programme is a European initiative for the implementation of information services dealing with environment and security, mainly based on observation data received from Earth Observation (EO) satellites. In the frame of this programme, ESA launched the Copernicus Sentinel-2A and Sentinel-2B optical imaging satellites, which are fully operating since June 2017. They are equipped with optical imaging sensor MSI (Multi-Spectral Instrument) acquiring optical data products with spatial resolution up to $10 \mathrm{~m}$ in 13 spectral bands from the VIS to the SWIR spectral domains ${ }^{1}$.

Sentinel-2 data are applied for a wide field of applications on land surface related to agriculture ${ }^{2,3}$, forestry ${ }^{4}$ and landcover change ${ }^{5}$. They are also used to monitor coastal and inland waters ${ }^{6,7}$. Most of these applications require an accurate atmospheric correction which is provided by Level-2A processor Sen2Cor. Sen2Cor is applied to correct monotemporal Sentinel-2 Level 1C products from the effects of the atmosphere in order to deliver radiometrically correct surface reflectance (SR) images. By-products are Aerosol Optical Thickness (AOT), Water Vapour (WV) and Scene Classification (SCL) maps ${ }^{8}$.

Image and Signal Processing for Remote Sensing XXVI, edited by Lorenzo Bruzzone, Francesca Bovolo

Emannuele Santi, Proc. of SPIE Vol. 11533, 1153304 - (c) 2020 SPIE

CCC code: $0277-786 \mathrm{X} / 20 / \$ 21 \cdot$ doi: $10.1117 / 12.2574035$ 


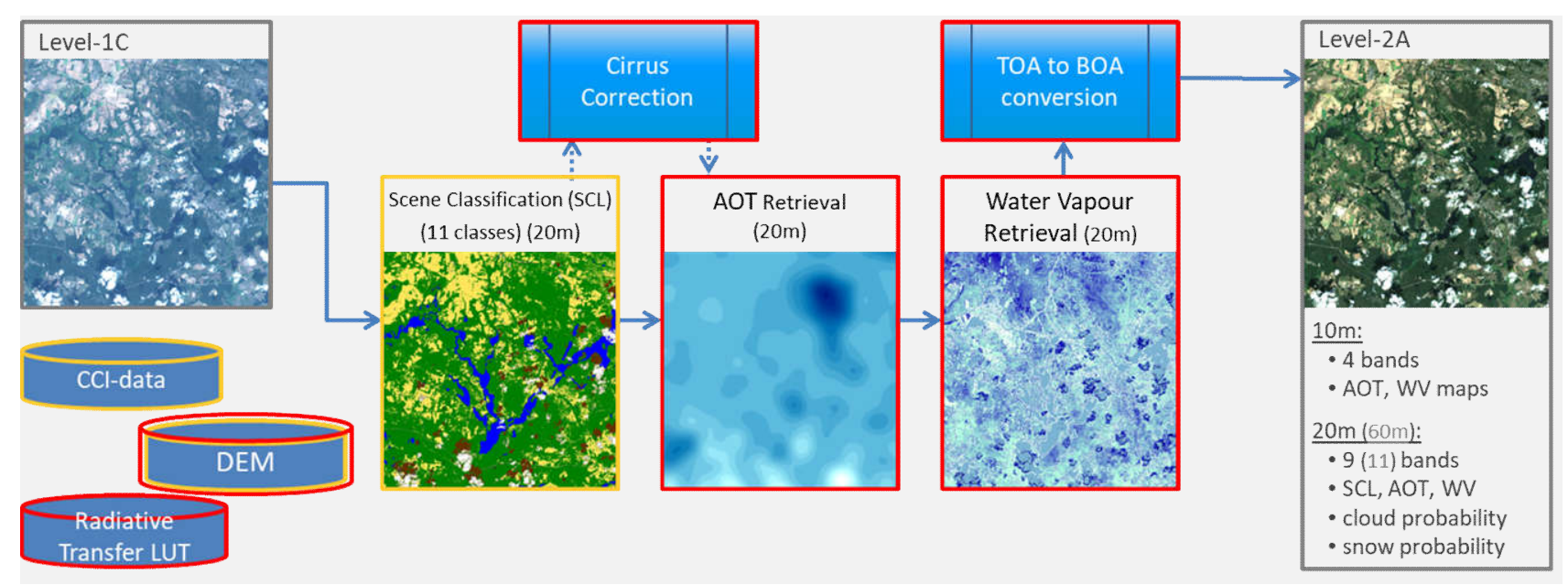

Figure 1: Sen2Cor processing chain. Yellow frames belong to scene classification and red frames belong to atmospheric correction modules.

Figure 1 shows the processing chain of Sen2Cor. Inputs to the processing are Sentinel-2 Level-1C data which are top-ofatmosphere (TOA) reflectances. The first processing step is scene classification (SCL) providing 11 classes in $20 \mathrm{~m}$ spatial resolution. SCL is based on a series of threshold tests on L1C spectral bands, band ratios and indices. ESA Climate Change Initiative (CCI) data and a Digital Elevation Model (DEM) are auxiliary data used for scene classification. Atmospheric correction modules are cirrus correction, aerosol optical thickness (AOT) and water vapour (WV) retrieval and last TOA to BOA (Bottom of Atmosphere) conversion. Auxiliary data used for atmospheric correction are the DEM and look-up-tables (LUT) of atmospheric radiation components precomputed with the libRadtran package ${ }^{9}$. LUTs were computed for Sentinel-2A and Sentinel-2B instrument spectral responses ${ }^{10}$.Cirrus correction is an optional pre-processing step after SCL is finished ${ }^{11}$. AOT retrieval is based on a dense dark vegetation algorithm $(\mathrm{DDV})^{12}$. The present fallback solution, if less than $2 \%$ DDV-pixels are found in the granule, is to perform the atmospheric correction with a pre-defined AOT. WV estimation is based on Atmospheric Pre-corrected Differential Absorption Algorithm (APDA) ${ }^{13}$. TOA to BOA conversion gives surface reflectance (SR) images. It includes terrain correction, correction for adjacency effects and an optional Bidirectional Reflectance Distribution Function (BRDF) correction $^{14}$. Final outputs of Sen2Cor are 9 bands of SR images at $20 \mathrm{~m}$ and 4 bands at $10 \mathrm{~m}$ spatial resolution together with AOT, WV, cloud probability and snow probability maps at $20 \mathrm{~m}$ spatial resolution. Sen2Cor can be configured to provide SR at $60 \mathrm{~m}$ spatial resolution including also bands 1 and 9 which are native $60 \mathrm{~m}$ bands and are not included in the $20 \mathrm{~m}$ product until Sen2Cor 2.8 .

Sentinel-2 Level-2A core products processed with Sen2Cor can be downloaded from Copernicus Open Access Hub ${ }^{15}$. Alternatively, Sen2Cor processor can be downloaded from ESA website as standalone tool for individual processing by the users ${ }^{16}$. It can also run as a plugin of the Sentinel-2 Toolbox (SNAP-S2TBX). Latest investigations of data quality provided by Sen2Cor processing are reported in the monthly L2A data quality reports ${ }^{17}$.

\section{SEN2COR HISTORY}

Sen2Cor is used by ESA since June 2017 for systematic L2A-processing of Sentinel-2 acquisitions over Europe. Global L2A-processing by Sentinel-2 ground segment (PDGS) started in March 2018. This ESA L2A core production gives the same results as can be obtained by the user if the same software version, auxiliary data and processing configuration are used. The only differences are related to product format, manifest generation, product quality metadata generation and JPEG2000 encoding library used. Users may observe differences due to DEM used. Whereas ESA L2A core production is generated with Planet $\mathrm{DEM}^{18}$, the "user" version has access to SRTM-DEM ${ }^{19}$ by default. Differences may also be observed because updates of Sen2Cor were not realized at the same time for the ESA L2A core production and for the public version of Sen2Cor (Figure 2). Most remarkable cases had been the use of CCI data package for scene classification and the LUT updates for the S2A spectral response functions version 3.0 ${ }^{10}$. The version history shows also that the archive of ESA L2A core products contains no homogenous time series. The only way to get a homogenous time series including data before May 2019 presently is to process the required data with the same Sen2Cor "user" version 
and configuration. Moreover, "user" processing gives the chance for processing with non-default configuration if necessary whereas ESA L2A core production can only be done with default configuration. Those non-default configurations could be switching on cirrus correction or increasing adjacency range for adjacency correction over dark water surrounded by forest. A reprocessing of the complete L2A archive is being discussed and prepared for collection 1 of ESA L2A core products archive.

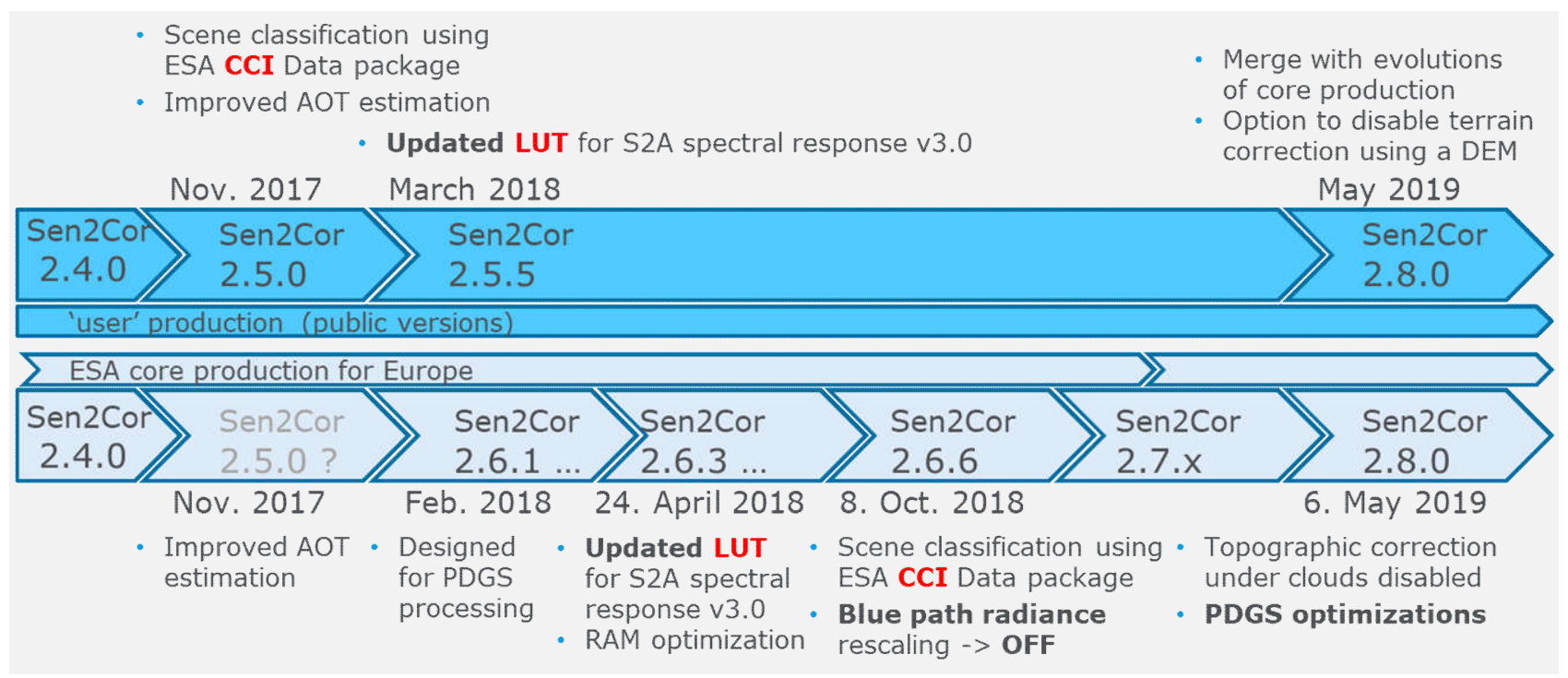

Figure 2: Sen2Cor version history (most important changes only); upper part = public (or “user") versions; lower part = versions used for ESA L2A core production

\section{SCENE CLASSIFICATION}

\subsection{SCL validation results}

Performance of scene classification was investigated comparing Sen2Cor classification outputs to an independent reference. Generation of this reference mask relied on visual inspection and labelling of individual pixels or polygons with help of different Sentinel-2 band composites, SR spectra, quality indicators, GoogleEarth [C2020 Google LLC] and the L1C data from cirrus band. Reference masks had been generated for 20 granules covering all continents, climate zones, seasons and land cover classes. They represent flat and mountainous terrain and different cloud types with cloudiness up to $62 \%$. Original Sen2Cor classification outputs were aggregated to clear classes (vegetated, not vegetated, water, snow or ice, unclassified), shadows (Dark features, cloud shadows) and clouds (cloud medium and high probability, thin cirrus). Note that this aggregation is not perfect due to class Dark features. This class contains topographic and cast shadows, however it can contain by present definition and decision tree burned areas or very dark water bodies and vegetation too. These all should belong to clear classes. This confusion is reflected in the validation results (Table 1) with $17 \%$ commission error of shadow classes due to classification of many clear pixels as shadow. On the other side there is also $28 \%$ omission of shadows for which the root cause may be applying a generic cloud top height distribution for cloud shadow detection. Overall classification accuracy of $94 \%$ together with low omission $(2.5 \%)$ und commission $(5.1 \%)$ of clear pixels are very good classification results. However, about 10000 cloud pixels classified as clear gives room for improvements. This misclassification is partly caused by the known problem of classification of bright objects as cloud. The larger part is due to pixels at cloud border which may be different interpreted by the classification algorithm and the person labelling the reference mask. The commission of clouds as clear pixels at cloud border can be reduced by a dilation of clouds which gives more reliability that pixels classified as clear are really clear. The consequence of this dilation is an increased omission of clear pixels. 
Table 1. Confusion matrix for SCL validation reporting number of validated pixels per aggregated class and resulting Producer Accuracy (PA), User Accuracy (UA), Omission Error (OE), Commission Error (CE) and Overall Accuracy (OA)

\begin{tabular}{|c|r|r|r|r|r|r|}
\hline & \multicolumn{1}{|c|}{ clear } & \multicolumn{1}{c|}{ shadows } & \multicolumn{1}{c|}{ clouds } & \multicolumn{1}{c|}{ UA } & \multicolumn{1}{c|}{ CE } \\
\hline clear & 287480 & 5080 & 10247 & & $94.9 \%$ & $5.1 \%$ \\
\hline shadows & 2611 & 13433 & 150 & $83.0 \%$ & $17.0 \%$ \\
\hline clouds & 4908 & 165 & 47859 & $90.4 \%$ & $9.6 \%$ \\
\hline & & & & & & \\
\hline PA & $97.5 \%$ & $71.9 \%$ & $82.2 \%$ & & OA & \\
\hline OE & $2.5 \%$ & $28.1 \%$ & $17.8 \%$ & & $94 \%$ & \\
\hline
\end{tabular}

\subsection{SCL outlook on the planned updates}

Following updates of SCL algorithm are in preparation for Sen2Cor version 2.10:

- Improved casted shadow algorithm

- Using snow climatology to reduce snow commission errors in high altitude clouds

- Update of cloud shadow algorithm with cloud top height estimation

- Inclusion of cloud / cloud shadow / snow dilation

- Bright isolated pixels identification

- Redefinition of class 'dark features' to contain only topographic and cast shadows

\section{AOT AND WV}

\subsection{AOT and WV validation results}

Validation of AOT and WV retrieval is realized by direct comparison of Sen2Cor outputs with sunphotometer measurements of AERONET sites ${ }^{20}$. Whereas AOT from AERONET is interpolated to $550 \mathrm{~nm}$ and time averaged over \pm 15 min to Sentinel-2 overpass time, the Sen2Cor outputs are spatially averaged over $9 \times 9 \mathrm{~km}^{2}$ area around the sunphotometer. Only clear land surface pixels are used for validation of AOT- and WV retrieval.
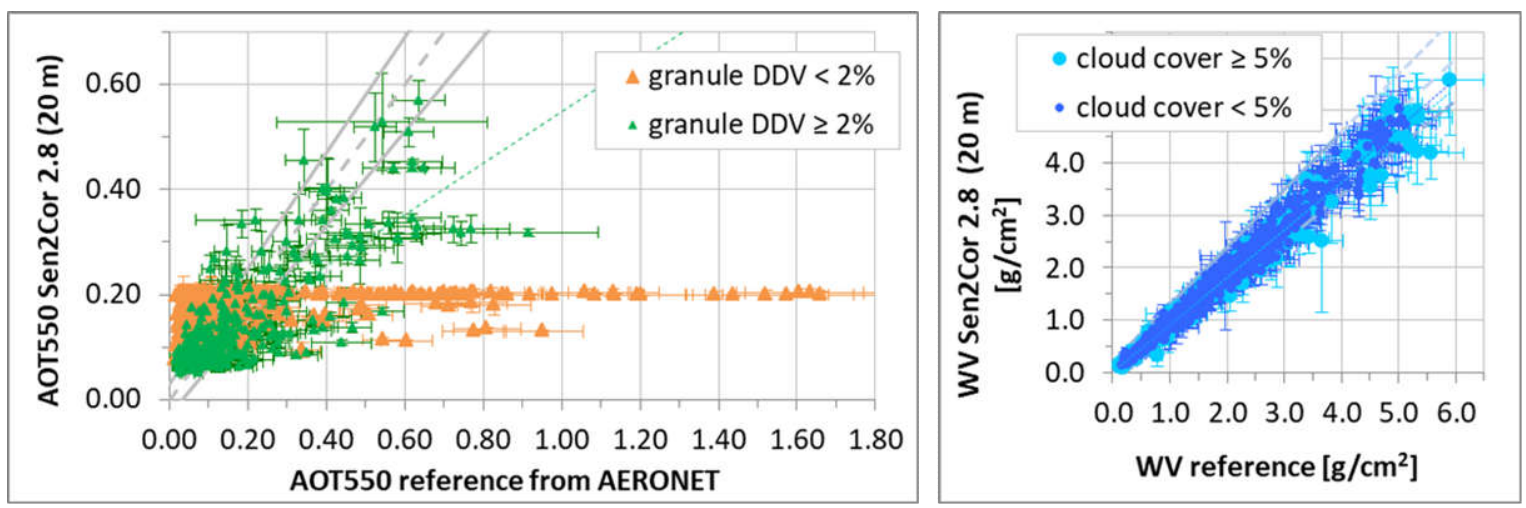

Figure 3: Left: Correlation plot of $\mathrm{AOT}_{550}$ retrieval of Sen2Cor over reference value from AERONET. Green triangles represent the results for AOT estimation with DDV algorithm and orange triangles show the results of fallback processing if the granule contains less than 2\% DDV pixels. Dashed line indicates retrieval equal to reference and solid lines mark the specification $\left|\Delta \mathrm{AOT}_{550}\right| \leq 0.1 * \mathrm{AOT}_{550}{ }^{\mathrm{ref}}+0.03$

Right: Correlation plot of WV retrieval of Sen2Cor over reference value from AERONET. Light blue circles represent the results for cloudy granules and dark blue circles show the results of granules with less than $5 \%$ cloud cover. Dashed line indicates retrieval equal to reference and solid lines mark the specification $|\Delta \mathrm{WV}| \leq 0.1 * \mathrm{WV}$ ref +0.2 . 
AOT retrieval clearly shows the need to have a better fallback solution if the granule does not contain enough reference pixels (Figure 3). Present fallback solution is processing with a fixed, pre-defined AOT-value which is about 0.2 at sea level. AOT can become smaller due to elevation of the site and due to a loop in Sen2Cor which reduces AOT in case that SR retrieval with the present AOT gives more than $1 \%$ of pixels with negative SR. Uncertainty of AOT retrieval is $\pm 0.24, \pm 0.11$ and \pm 0.29 for complete data set, DDV subset and subset of granules processed with fallback solution respectively. ESA L2A core production and user production with the public version of Sen2Cor don't give identical numbers due to differences in the DEM used and different patches implemented. However, these differences are at the third digit after decimal point and thus not significant.

WV retrieval is very accurate with uncertainty of $\pm 0.24 \mathrm{~g} / \mathrm{cm}^{2}$. It shows light underestimation with larger differences occurring for cloudy granules than for nearly cloudless. Agreement between ESA L2A core product and user product is nearly complete.

\subsection{AOT retrieval planned update}

An update of the AOT-retrieval fallback solution is prepared for Sen2Cor version 2.10. Copernicus Atmospheric Monitoring Service (CAMS) ${ }^{21}$ data will be used to provide AOT at $550 \mathrm{~nm}$ in case that Sen2cor cannot retrieve AOT due to lack of DDV-pixels ${ }^{22}$. The main effect is replacing the strong outliers visible in Figure 3 by more reasonable values leading to a strong reduction of AOT dispersion. Performance of the update was tested with running Sen2Cor on a test data set of granules without DDV-pixels with identical configuration except the fallback solution used. Uncertainty of $\mathrm{AOT}$ at $550 \mathrm{~nm}$ reduced from 0.35 for processing with the present fallback solution to 0.19 using the CAMS-AOT.

\section{SURFACE REFLECTANCE}

\subsection{SR validation results}

The amount of available reference measurements for validation of SR is very limited. SR measurements at RADCALNET $^{23}$ sites LaCrau and Gobabeb had been used as reference data for Atmospheric Correction Intercomparison eXercise $^{24}$. Left side of Figure 4 shows a summary of SR retrieval performance for this data set. Sen2Cor is almost within $\pm 2 \%$ of measured reference data except bands 5 and 11 . Note that no reference measurements are available for bands 1 and 12. Right side of Figure 4 compares SR retrieval of Sen2Cor with own reference measurements for vegetated sites. Shape of spectra is retrieved very well with Pearson correlation coefficient higher than 0.99. Sen2Cor little under corrects with root mean square difference RMSD between 0.015 and 0.025 . Difference Sen2Cor minus reference is shown in the plot with the green dotted line on the bottom. The worse behavior of bands 5 and 11 found for the most dry sites LaCrau and Gobabeb cannot be found for the three given examples at vegetated sites. This differing result has to be investigated in more detail. Right side of Figure 4 also compares SR obtained from the ESA L2A core product with the user product. Differences between both are near to zero and at least one magnitude smaller than differences between Sen2Cor-output and measured reference. Core product and user product give equivalent SR spectra.
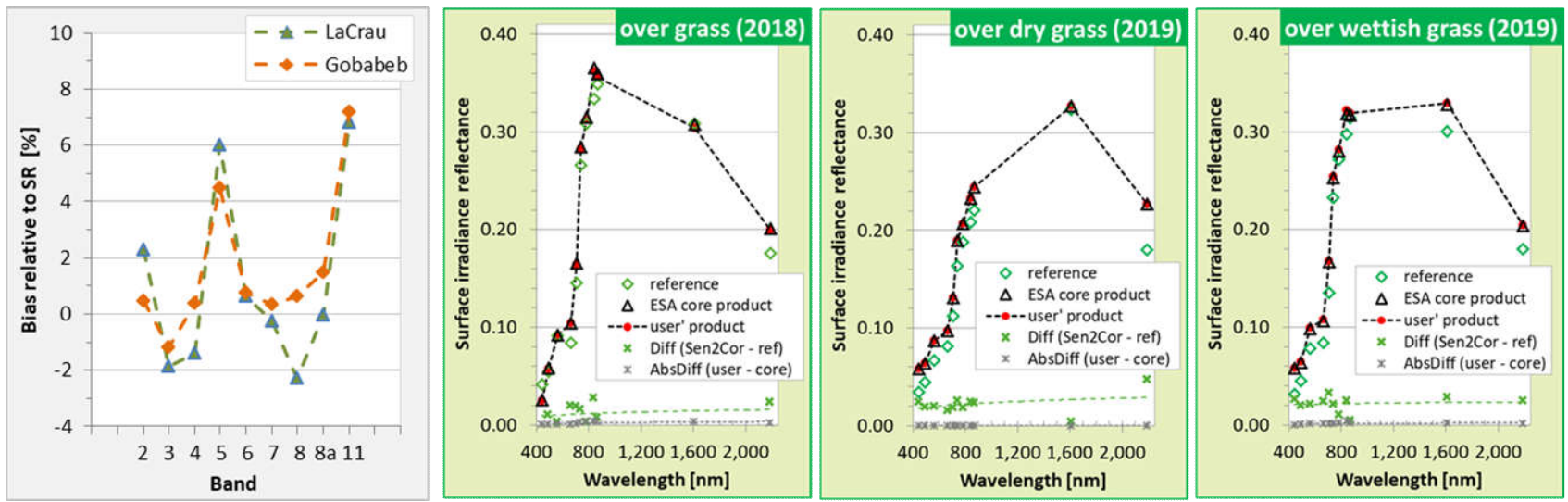

Figure 4: Left: Average relative bias per band of Sen2Cor SR retrieval compared with ground based measurements at RADCALNET test sites LaCrau and Gobabeb as reference. Right: Comparison of SR spectra obtained from Sen2Cor with reference measurements over vegetated sites. 


\section{CONCLUSIONS AND OUTLOOK}

Reported investigations confirm good performance of Sen2Cor for all of scene classification, WV retrieval and SR estimation. Several updates of the processor are on the way to improve some data quality weaknesses found. It was shown, that ESA L2A core product gives equivalent results to 'user' products obtained with the public available Sen2Cor. ESA L2A core products are the easiest way to get atmospheric corrected SR products. However, they are based on a default configuration. 'User' product gives the opportunity to process with non-default configuration, which may have advantages and give better results in some situations than relying on default configuration. ESA archive of Sentinel-2 L2A-products has still no 'collection' providing a (long) homogenous time series until the archive is reprocessed. 'User' processing gives the opportunity to generate a homogenous time series for an area of interest.

Main updates resp. product evolutions foreseen for Sen2Cor are summarized in Figure 5. Sen2Cor 2.9 will provide some processing fixes. Sen2Cor 2.10 will contain several scene classification improvements, it will support the usage of CAMS data for AOT fallback and Copernicus DEM. It will support changes of the product structure corresponding to a new Product Specification Document (PSD) like output of band 1 in the $20 \mathrm{~m}$ spatial resolution product and extending the product with quality indicators with regard to L2A processing. Sen2Cor 3.0 will be migrated to Python 3.7 and will support processing of Landsat-8 data.

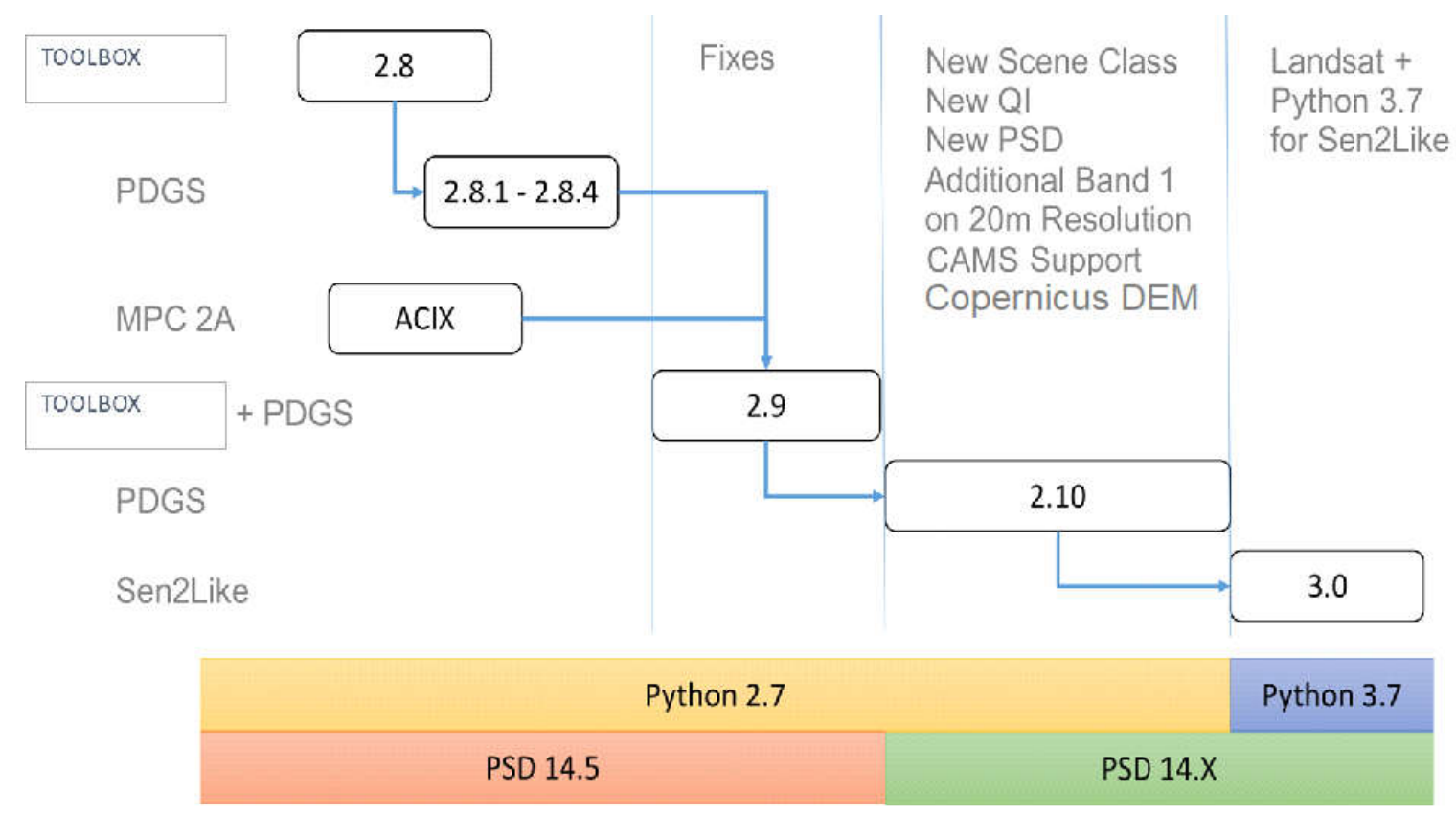

Figure 5: Overview over L2A product evolutions on the way

\section{ACKNOWLEDGMENTS}

The research was performed as part of the Copernicus Sentinel-2 Mission Performance Center activities which are managed by ESA. The authors thank the PI investigators and their staff for establishing and maintaining the AERONET sites used in this investigation. 


\section{REFERENCES}

[1] Drusch, M., et al., "Sentinel-2: ESA's Optical High-Resolution Mission for GMES Operational Services," Remote Sensing of Environment 120, 25-36 (2012).

[2] Defourny, P., et al., "Near real-time agriculture monitoring at national scale at parcel resolution: Performance assessment of the Sen2-Agri automated system in various cropping systems around the world," Remote Sensing of Environment 221, 551-568 (2019).

[3] Bontemps, S., et al., "Sentinel-2 for Agriculture": Supporting Global Agriculture Monitoring," IEEE International Geoscience and Remote Sensing Symposium (IGARSS), 4185-4188 (2015).

[4] Immitzer, M., et al., "Optimal Input Features for Tree Species Classification in Central Europe Based on MultiTemporal Sentinel-2 Data," Remote Sensing, 11(22), 1-23 (2019) < http://creativecommons.org/licenses/by/3.0/ \%U https://www.mdpi.com/2072-4292/11/22/2599 >.

[5] Gedefaw, A.A., et al., "Analysis of Land Cover Change Detection in Gozamin District, Ethiopia: From Remote Sensing and DPSIR Perspectives," Sustainability 12(11), 1-25 (2020).

[6] Du, Y., et al., "Water Bodies' Mapping from Sentinel-2 Imagery with Modified Normalized Difference Water Index at 10-m Spatial Resolution Produced by Sharpening the SWIR Band," Remote Sensing 8(4), 1-19 (2016).

[7] Ansper, A. and K. Alikas, "Retrieval of Chlorophyll a from Sentinel-2 MSI Data for the European Union Water Framework Directive Reporting Purposes". Remote Sensing, 11(1), 1-26 (2019).

[8] Louis, J., B. Pflug, and Sentinel-2 MPC Team, "Level-2A Algorithm Theoretical Basis Document," (2020).

[9] Emde, C., et al., "The libRadtran software package for radiative transfer calculations (version 2.0.1)". Geoscientific Model Development 9(5), 1647-1672 (2016).

[10] European Space Agency, "Sentinel-2 Spectral Response Functions (S2-SRF), 2017, COPE-GSEG-EOPG-TN15-0007; Version 3.0,", < https://sentinels.copernicus.eu/web/sentinel/user-guides/sentinel-2-msi/documentlibrary/-/asset_publisher/Wk0TKajiISaR/content/sentinel-2a-spectral-responses > (11.06.2020).

[11] Richter, R., et al., "Correction of cirrus effects in Sentinel-2 type of imagery," International Journal of Remote Sensing 32(10), 2931-2941 (2011).

[12] Kaufman, Y.J., et al., "The MODIS 2.1-mu m channel - Correlation with visible reflectance for use in remote sensing of aerosol," Ieee Transactions on Geoscience and Remote Sensing 35(5), 1286-1298 (1997).

[13] Schlapfer, D., et al., "Atmospheric precorrected differential absorption technique to retrieve columnar water vapor," Remote Sensing of Environment 65(3), 353-366 (1998).

[14] Richter, R., "Correction of satellite imagery over mountainous terrain" Applied Optics 37(18), 4004-4015 (1998).

[15] European Space Agency, “Copernicus Open Access Hub,” ＜https://scihub.copernicus.eu/dhus/\#/home $>$ (24. August 2020).

[16] European Space Agency, "step, science toolbox exploitation platform, Sen2Cor," $<$ http://step.esa.int/main/thirdparty-plugins-2/sen2cor/ > (24. August 2020).

[17] Sentinel-2 MPC Team, "Sentinel-2 L2A Data Quality Report (S2-PDGS-MPC-L2ADQR)," $<$ https://earth.esa.int/web/sentinel/user-guides/sentinel-2-msi/document-library/> (18 August 2020).

[18] Planet Observer, "Planet DEM," < https://www.planetobserver.com/products/planetdem/planetdem-30/ . > (25 September 2018).

[19] Jarvis, A., et al., "Hole-filled SRTM for the globe Version 4," Slate, 2008, Available from: available from the CGIAR-CSI SRTM 90m Database < http://srtm.csi.cgiar.org >) (24 August 2020).

[20] Sinyuk, A., et al., "The AERONET Version 3 aerosol retrieval algorithm, associated uncertainties and comparisons to Version 2," Atmospheric Measurement Techniques 13(6), 3375-3411 (2020).

[21] ECMWF, "Copernicus Atmosphere Monitoring Service," < http://atmosphere.copernicus.eu/ $>$ (9. February 2018).

[22] Louis, J., et al., "Integration and Assimilation of Meteorological (Ecmwf) Aerosol Estimates into Sen2cor Atmospheric Correction," IEEE International Geoscience and Remote Sensing Symposium (IGARSS), 18941897 (2018).

[23] Bouvet, M., et al., "RadCalNet: A Radiometric Calibration Network for Earth Observing Imagers Operating in the Visible to Shortwave Infrared Spectral Range," Remote Sensing 11(20), 1-24 (2019).

[24] Vermote, E., et al., "ACIX - Atmospheric Atmospheric Correction Inter-comparison eXercise,” IEEE International Geoscience and Remote Sensing Symposium (IGARSS) 8493-8496 (2019). 